\title{
Automated Sample Processing for Flow Cytometry
}

\author{
Jasenka Memisevic, Lisa C. Shriver-Lake, Peter B. Howell Jr., Joel P. Golden, Nastaran Hashemi, Kirsten B. Jackson, \\ Frances S. Ligler \\ Center for Bio/Molecular Science and Engineering \\ U.S. Naval Research Laboratory \\ Washington, DC, USA \\ frances.ligler@nrl.navy.mil
}

\begin{abstract}
The utility of pathogen and toxin detection systems depends not only upon sensitivity, specificity and capability for multiplexed recognition, but also on access at the point of need, ease of use, and response time. Combining microfluidics and optical biosensors facilitates miniaturization and automation, while careful design makes it possible to test variable quantities of complex matrices such as food $(\mathrm{mL})$ and clinical samples $(\mu \mathrm{L})$. We report on progress towards an optofluidic system that combines a rotating magnet trap for automated sample processing and a microflow cytometer capable of 4-color analysis to achieve multi-analyte diagnostics. Sample and antibody-coated magnetic beads are introduced into a moving magnetic field created by magnets rotating in the opposite direction of the flow. The color-coded magnetic beads with captured target remain in suspension while fluorescent tracer reagents are introduced to the flow, thus optimizing the binding kinetics and minimizing aggregation. Magnetic beads with bound target and tracer reagents are released for multiplexed analysis by reversing the direction of spinning magnets.
\end{abstract}

\section{INTRODUCTION}

The prudent incorporation of microfluidics into biosensors can facilitate interrogation of complex matrices, such as food and clinical samples, given a configuration resistant to clogging problems. More importantly, microfluidics can provide technology for automated operations within a portable system, not only by miniaturizing the sensor component but also by providing a tool for on-chip sample preparation. Performance of assays inside a microfluidic chip requires that sample processing times and reagent additions are carefully controlled. Additionally, there is frequently a need to concentrate the target analytes to achieve required detection sensitivity.

Immunomagnetic separation has been used extensively to concentrate pathogens. Several investigators have used static magnets with microfluidics to separate beads from solution [14]. In one example, a microfluidic channel is utilized to introduce magnetic beads into the analysis region of magnetic device. The beads are then drawn across the channel by the magnetic field, passing through laminar streams containing assay reagents. Alternatively, the beads are passed by a permanent magnet between different micro-compartments, each holding a different reagent. The magnetic beads are first concentrated into a bolus, and then moved from one compartment to the next in bulk.

A rotating magnetic trap with strategically spaced magnets has the advantage of both pre-concentrating the magnetic beads and also mixing the beads with the assay reagents. The ability to mix beads in microchannel during an assay both exposes more bead surface to the reagent and improves binding kinetics. The use of rotating magnets for capture and release has been reported by Anderson et.al. [5] for capture of peptides on antibody-coated magnetic beads in a loop of tubing prior to elution of the peptides for mass spectrometry. We report on the development and characterization of a rotating magnetic trap coupled to a microfluidic channel for performing multiplexed biological assays. The magnetic trap (MagTrap) has the capability to concentrate, mix, and subsequently release the beads. This device notes progress towards a fully automated sample processing component for automated biosensor analyses.

\section{MATERIALS AND METHODS}

\section{A. Materials}

Assays were performed using Luminex MagPlex carboxylated magnetic spheres (Luminex Corp, Austin, TX). Proteins immobilized to the beads' surfaces included $\alpha-E$. coli IgG (KPL, Gaithersbug, MD), chicken IgY (Jackson Immunoresearch Labs, West Grove, PA), and bovine serum albumin (BSA; Sigma Aldrich, St. Louis, MO). E. coli (KPL) was used as the target analyte, biotinylated anti-chicken IgY (Jackson Immunoresearch Labs) was the positive control, and BSA provided the negative control. Dilutions were done in deionized water or phosphate buffered saline containing 0.05 $\%$ Tween-20 (PBST, Sigma-Aldrich, St. Louis, MO) with $0.1 \%$ sodium azide (Sigma Aldrich,). PBSTB with $1 \mathrm{mg} / \mathrm{ml} \mathrm{BSA}$ (PBSTB) was used for all assays. Chemical surface modification of the MagPlex beads was achieved using 1-ethyl3-[3-dimethylaminopropyl]carbodiimide hydrochloride (EDC) with N-hydroxy sulfosuccinimide (Sulfo-NHS), both from Sigma-Aldrich, and the activation buffer was $0.1 \mathrm{M}$ sodium phosphate, $\mathrm{pH}$ 6.0). Fluorescence detection was done with streptavidin phycoerythrin (SAPE, Prozyme, San Leandro, CA).

\section{B. Preparation of COOH Magnetic Beads forMicoflow Assay}

Attachment of amine-containing molecules to $\mathrm{COOH}$ beads was done by following the protocol of Taitt et.al [6]. The capture antibody was attached to a bead set with distinct amounts of two fluorophores, and the bead identification was used later in the multiplexed analyses. Briefly, magnetic beads were washed $3 \mathrm{x}$ with activation buffer using a magnetic 


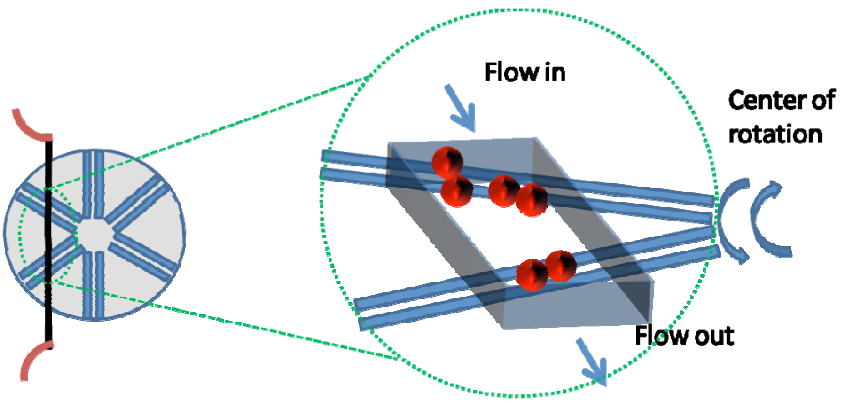

Figure 1. Schematic of the MagTrap device. On the left is the circular magnet holder with a microchannel overlayed. Magnication on the right shows the beads collecting in the magnetic fields, the flow direction, and the rotational directions available to the magnets for processing and release.

microcentrifuge tube holder. The EDC and sulfo-NHS $(50 \mathrm{mg} / \mathrm{ml}$ in deionized water for both) were added in equal volumes to the beads such that the final concentration was $5 \mathrm{mg} / \mathrm{ml}$. After incubation and washing of the linkers, the capture antibodies were added in $1 \mathrm{mg} / \mathrm{ml}$ concentrations in PBS, pH 7.4.

\section{MagTrap Sample Processing}

For demonstrating the operation of the MagTrap, mixed sets of magnetic beads were added to the sample containing $1 \times 10^{6}$ cells $/ \mathrm{ml} \mathrm{E}$. coli. After 20 minutes, the sample was introduced into the MagTrap to process the immunoassay along with the positive and negative controls. Magnets were rotated in the direction opposite to the fluid flow in microchannel, while the beads, at a concentration of 50 beads/ $\mu$ l of each type, were trapped and concentrated (Figure 1). The tracer cocktail, including $10 \mu \mathrm{g} / \mathrm{ml}$ of biotinylated $\alpha-E$. coli, $750 \mathrm{ng} / \mathrm{ml}$ biotinylated sheep $\alpha$-chicken IgY, and $10 \mu \mathrm{g} / \mathrm{ml} \mathrm{BSA}$, was passed through the channel for 5 minutes, followed by PBSTB for 3 minutes, and $7.5 \mu \mathrm{g} / \mathrm{ml} \mathrm{SAPE}$ for 3 minutes to complete the sandwich immunoassay. PBSTB was added during the release step. Flow was maintained throughout the processing procedure at $10 \mu \mathrm{l} / \mathrm{min}$ and a linear velocity of $150 \mathrm{~mm} / \mathrm{min}$. The microchannel was $6 \mathrm{~cm}$ long and had a trapezoidal cross section, with a $130 \mu \mathrm{m}$ height, base 1 of $500 \mu \mathrm{m}$ and base 2 of $355 \mu \mathrm{m}$.

Sample processing under flow in the MagTrap was compared to that in a conventional static assay. Processing time and reagent concentrations were identical in both methods. The conventional assay had static incubations in a $1.5 \mathrm{ml}$ reaction tube and reagent solutions were added by holding the magnetic beads in place by a stationary magnetic field.

\section{Data Collection and Analysis}

The MagTrap performance data consisted of sample collections during various events. The specific events were: passage of beads through microchannel without magnets, passage through channel during magnetic trapping, passage through channel during magnetic release and passage through channel after the magnets were physically removed from the microchannel. The Accuri C6 flow cytometer (Accuri Cytometers, Ann Arbor, MI) was used to determine the number of beads collected during each event, as well as in the stock sample of MagPlex beads. The effectiveness of trapping and release by the MagTrap was evaluated by determining the bead/ $\mu$ l concentrations of MagPlex beads collected during passage through each stage, normalized to the concentration passing through channel before exposure to magnets.

Assay data was collected using the NRL Luminex 100 and analyzed with the xPONENT software (Luminex Corporation, Austin, TX). The Luminex 100 utilizes sheath flow to hydrodynamically focus the sample solution. As samples pass through the interrogation region, the beads' internal dyes (bead identification) and phycoerythrin (presence of antigen) are excited and their fluorescence emission spectra were detected. Analysis with XPONENT identified the beads in sample and provided information about the median and mean phycoerythrin fluorescence, count of events and \% coefficient of variance associated with each bead type. The PE fluorescence of E.coli -labeled beads was compared to those of positive and negative, chicken and BSA, respectively. The fluorescence data was correlated to bead count in each group, thus normalizing fluorescence intensity to number of beads.

\section{RESULTS AND DISCUSSION}

\section{A. Trapping and Release Efficiency of the MagTrap}

Initial experiments were designed to confirm that (1) the rotating magnets could trap the weakly magnetic beads in the flowing stream and (2) that reversing the magnets instead of removing them all together was sufficient to release the beads from the MagTrap. Fig. 2 represents a comparison of magnetfree and magnet-assisted microsphere release, after magnetic trapping. The beads were trapped over an 18-minute period and samples were collected in three-minute intervals, before the magnets were either physically removed away from the microchannel, thus causing the beads to freely exit the channel with the aid of fluid flow, or released by reversing the rotation of the magnets to match the direction of the flow.

Results shown in Fig. 2 suggest that the magnet-assisted microsphere release was more effective than the magnet-free release. While approximately equal quantities of beads were released using both methods, the magnet-assisted release was more efficient at removing all beads at once. In the magnetfree release, the beads were removed in three steps, while with presence of magnets removal was carried out in a single step.

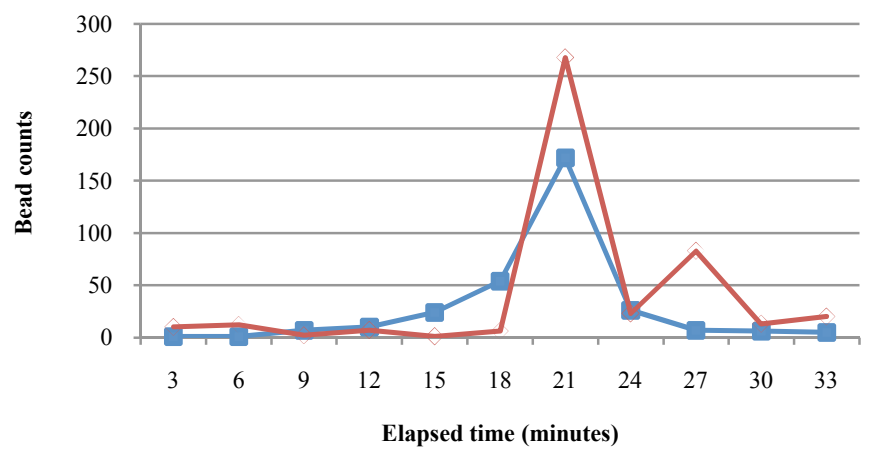

Figure 2. Bead capture efficiency of the MagTrap with a)magnet-free release $\left.(--\rangle_{--}\right)$and b) magnet-assisted release (-- --). Samples were collected over three-minute intervals. Bead capture took place during the first 18 minutes, after which time the magnets were removed or reversed to release the beads. 


\section{B. MagTrap Fluid and Conventional Static Assays}

The device was assessed through immunoassay performance. Magnetic beads were labeled with antibody against $E$. coli, with chicken IgY as a positive control and with BSA as a negative control. After 20-minute incubation with the $E$. coli antigen, beads were loaded into the microchannel. The magnetic trap held the beads inside the channel, while mixing them using the opposing forces generated by the rotating magnetic fields and the flow.

Table 1 shows results produced by processing using the MagTrap compared to conventional static processing. In the MagTrap, the magnetic beads were moved against the flow stream using magnetic fields during the reagent exposure. In the conventional static method, the beads were incubated with reagents and then held in the tube by a stationary magnet while solutions were changed. The median fluorescence of phycoerythrin associated with each bead was measured using the Luminex 100.

Results indicate that the assays performed under flow were successful. The negative control BSA beads exhibited negligible fluorescence in comparison to the positive control beads with chicken IgY. The $E$. coli fluorescence was even higher than the positive control, indicating that the antibodybound cells were not sheared off the beads during processing in the magnetic trap. Moreover, the magnetic bead trap was effective at retaining the beads, and 5 minutes exposure to the antibody tracers and 3 minutes for the SAPE label were sufficient incubation times for reagent binding.

Similar fluorescence results were obtained in both methods, indicating that the MagTrap processed the beads as efficiently as manual mixing and reagent addition. However, the bead counts were lower in the microflow system due to the beads sticking to channel walls.

\section{Static Assays in Microflow Cytometer}

The next step of this work will involve uniting the MagTrap with a microfluidic cytometer for a total analysis system. The Microflow Cytometer was developed in house and consists of a hydrodynamically focused sample core that passes through an optical interrogation window. At the interrogation window, the dyes used to code the beads and the SAPE fluorescent tag are excited using $532 \mathrm{~nm}$ and $635 \mathrm{~nm}$ lasers. Fluorescence emission and side scatter spectra were

TABLE I. PERFormanCE UNDER Flow AND STATIC CONDITIONS

\begin{tabular}{|c|c|c|c|}
\hline & \multicolumn{3}{|c|}{ Median PE fluorescence } \\
\hline & BSA & Chicken & E.coli \\
\hline $\begin{array}{c}\text { Microfluidic Assay } \\
\text { (flow) }\end{array}$ & 68 & 16,000 & 18,000 \\
\hline $\begin{array}{c}\text { Conventional Assay } \\
\text { (static) }\end{array}$ & 4.3 & 19,000 & 15,000 \\
\hline
\end{tabular}

collected using four PMTs. Current results, as reported by Kim et al [7], show positive bead identifications with nonmagnetic coded beads. Detection with a 12-plex assay was achieved, including E.coli, chicken and BSA, with the Microflow Cytometer. In the future, we will integrate the MagTrap with the Microflow Cytometer for a fully automated multiplexed biosensor.

\section{CONCLUSIONS}

An automated sample processing device has been demonstrated that uses a rotating magnetic field to move magnetic target-capture beads in the direction opposite to the flow in a microchannel. After bead concentration from the initial sample, the beads are exposed to successive reagent streams with efficient mixing. The processed beads are efficiently released when the rotation of the magnets is reversed. Additionally, the binding is as efficient in the flow (or more so) as that obtained using conventional manual processing.

\section{ACKNOWLEDGMENTS}

$\mathrm{JM}$ is a postdoctoral fellow of the National Research Council and NH is a fellow is a fellow of the American Society of Electrical Engineering. KBJ was a Naval Research Enterprise Internship Program summer intern. The work presented here was performed under NIH grant UO1 A1075489 and ONR/NRL 6.2 work unit 6336. The views presented here are those of the authors and do not represent the opinion or policy of the National Institutes of Health, Department of Health and Human Services, the US Navy, or the Department of Defense.

\section{REFERENCES}

[1] M. Shikida and et al., "A palmtop-sized rotary-drive-type biochemical analysis system by magnetic bead handling," Journal of Micromechanics and Microengineering, vol. 18, p. 035034, 2008.

[2] J. J. Lai, et al., "Dynamic bioprocessing and microfluidic transport control with smart magnetic nanoparticles in laminar-flow devices," $L a b$ on a Chip, vol. 9, pp. 1997-2002, 2009.

[3] S. A. Peyman, et al., "Mobile magnetic particles as solid-supports for rapid surface-based bioanalysis in continuous flow," Lab on a Chip, vol. 9, pp. 3110-3117, 2009.

[4] F. Lacharme, et al., "Full on-chip nanoliter immunoassay by geometrical magnetic trapping of nanoparticle chains," Analytical Chemistry, vol. 80, pp. 2905-2910, Apr 152008.

[5] N. L. Anderson, et al., "SISCAPA Peptide Enrichment on Magnetic Beads Using an In-line Bead Trap Device," Molecular \& Cellular Proteomics, vol. 8, pp. 995-1005, May 2009.

[6] C. R. Taitt, et al., "Surface Modification and Biomolecule Immobilization on Polymer Spheres for Biosensing Applications," in Biomedical Nanotechnology. vol. 726, S. J. J. Hurst, Ed., ed: Humana Press, 2011, pp. 77-94.

[7] F. S. Ligler and J. S. Kim, The Microflow Cytometer. Singapore: Pan Stanford Publishing, 2010. 